\title{
Associations between diet quality and socio-economic factors in the UK population
}

Diet Quality Indices (DQIs) assess the extent to which individuals' overall diet conforms to dietary guidelines. A DQI originally developed in 2009 to score diets in line with the then Scottish Dietary Targets ${ }^{(1)}$, has recently been updated in line with the current Scottish Diet Goals $^{(2)}$. The aim of the present study was to investigate the association of this updated DQI with socio-economic factors, in the UK population from years 1-6 (2008-2014) of the National Diet and Nutrition Survey. A score (0-10) was assigned to each of the 9 DQI components (fruit and vegetables, oily fish, red and processed meat, total fat, saturated fat, total carbohydrates, free sugars, fibre and energy density) then summed and adjusted to a percentage score. Associations between DQI score and socio-economic factors were examined in those aged 2+ years adjusting for the other socio-economic variables, survey year, country, sex, age, ethnicity, number of people in household and children in household.

Mean DQI percentage scores were low overall (mean 41.5; $95 \%$ CI 40.9, 42.0) and in all subgroups. DQI was significantly related to house ownership and NS-SEC classification with those owning their own home and those in managerial and professional occupations having the highest DQI. Although household income and work status were related to DQI score in univariate analyses (results not shown), when adjusting for other factors they were no longer significant. There were clear associations between diet quality and house ownership and occupation in the UK population between 2008-2014.

\begin{tabular}{|c|c|c|c|c|c|}
\hline & & & $\begin{array}{r}\text { Diet } \\
\text { per }\end{array}$ & $\begin{array}{l}\text { ity Index } \\
\text { ge score }\end{array}$ & \\
\hline & & $\mathrm{n}^{*}$ & Mean & $95 \% \mathrm{CI}$ & $\mathrm{P} * *$ \\
\hline Equivalised household income quintile & 1 (lowest) & 1528 & $44 \cdot 4$ & $42 \cdot 4,46 \cdot 4$ & \\
\hline & 2 & 1541 & $46 \cdot 4$ & $44 \cdot 3,48 \cdot 6$ & \\
\hline & 3 & 1510 & $46 \cdot 3$ & $44 \cdot 1,48 \cdot 4$ & \\
\hline & 4 & 1585 & $46 \cdot 3$ & $44 \cdot 1,48 \cdot 5$ & \\
\hline & 5 (highest) & 1533 & $46 \cdot 5$ & $44 \cdot 2,48 \cdot 8$ & $0 \cdot 121 \dagger$ \\
\hline House ownership & Own outright & 1599 & $48 \cdot 9$ & $46 \cdot 7,51 \cdot 1$ & Reference \\
\hline & Own with mortgage & 3467 & $47 \cdot 3$ & $45 \cdot 3,49 \cdot 3$ & 0.085 \\
\hline & Rent local authority & 899 & $42 \cdot 8$ & $40 \cdot 4,45 \cdot 2$ & $<0.001$ \\
\hline & Rent housing association & 580 & $44 \cdot 7$ & $42 \cdot 1,47 \cdot 3$ & $0 \cdot 001$ \\
\hline & Rent privately, furnished & 194 & $46 \cdot 3$ & $42 \cdot 5,50 \cdot 0$ & $0 \cdot 188$ \\
\hline & Rent privately, unfurnished & 958 & $45 \cdot 7$ & $43 \cdot 5,48 \cdot 0$ & $0 \cdot 006$ \\
\hline Work status & In full or part-time employment & 2379 & $46 \cdot 0$ & $43 \cdot 7,48 \cdot 4$ & Reference \\
\hline & Not working at present & 2243 & $46 \cdot 4$ & $44 \cdot 1,48 \cdot 8$ & 0.639 \\
\hline & Going to school or college full-time & 3075 & $45 \cdot 4$ & $42 \cdot 9,47 \cdot 9$ & 0.691 \\
\hline NS-SEC classification (household reference person) & Higher managerial and professional occupations & 1171 & $47 \cdot 2$ & $45 \cdot 0,49 \cdot 5$ & Reference \\
\hline & Lower managerial and professional occupations & 1973 & $46 \cdot 4$ & $44 \cdot 4,48 \cdot 4$ & $0 \cdot 340$ \\
\hline & Intermediate occupations & 760 & $45 \cdot 0$ & $42 \cdot 6,47 \cdot 5$ & $0 \cdot 062$ \\
\hline & Small employers and own account workers & 776 & $44 \cdot 0$ & $41 \cdot 6,46 \cdot 4$ & $0 \cdot 004$ \\
\hline & Lower supervisory and technical occupations & 732 & $44 \cdot 3$ & $41 \cdot 8,46 \cdot 8$ & $0 \cdot 016$ \\
\hline & Semi-routine occupations & 1077 & $45 \cdot 3$ & $43 \cdot 0,47 \cdot 6$ & $0 \cdot 089$ \\
\hline & Routine occupations & 876 & $44 \cdot 0$ & $41 \cdot 5,46 \cdot 5$ & $0 \cdot 009$ \\
\hline & Never worked & 205 & $45 \cdot 1$ & $41 \cdot 8,48 \cdot 5$ & $0 \cdot 236$ \\
\hline & Other & 127 & $52 \cdot 4$ & $46 \cdot 5,58 \cdot 2$ & $0 \cdot 095$ \\
\hline
\end{tabular}

*unweighted $n .{ }^{* *}$ Multivariate GLM applying sampling weighting variables. ${ }^{\dagger} P$ for linear trend.

This work was supported by funding from the Scottish Government's Rural and Environment Science and Analytical Services (RESAS) division.

1. Armstrong J, Sherriff A, Wrieden WL, et al. (2009) Deriving and Interpreting Dietary Patterns in the Scottish Diet: Further Analysis of the Scottish Health Survey and the Expenditure and Food Survey. Food Standards Agency Scotland.

2. The Scottish Government (2016) Revised Dietary Goals for Scotland - March 2016. 